\title{
An Efficient Data Structure for Querying XML Document in Maritime Field
}

\author{
Chia-Hung SHIH $^{*}$, Nobukazu WAKABAYASHI ${ }^{* *}$ \\ Saburo YAMAMURA Y** $^{* *}$ and Juri HARADA HA** $^{* * *}$
}

\begin{abstract}
Nowadays, data storage methods have been gone in the direction of integration with the internet structure. One of the most popularized data storage technologies is relational database. In the contrast, along with the coming of Business to Business (B2B) generation, the tendency of using Entensible Markup Language (XML) document as data exchange format recently becomes more and more evident. XML data model could be a better or suitable solution for the maritime field because ships offshore are not able to access or update database through the internet in real time.

Compared with relational database, XML has the semantic advantage. This characteristic allows it to communicate with servers and to achieve the objective of automatic operations. On the other hand, because XML is built in pure text format, compared with the binary structure of database, XML takes more data storage space and has obvious backward query efficiency. In our research, in order to reduce the disadvantage of XML essence, the data storage structure for ship data modeling is investigated from query efficiency view and we apply this data model on the container operation as a sample to verify the improvement of query efficiency.
\end{abstract}

Keywords : XML, Query efficiency, Data structure, Duplication of data

\section{Introduction}

In our previous research, we proposed a XML ship data model for fleet management ${ }^{(1)}$. This data model can be operated independently within the ship, and when the ship arrive at port, it can be integrated with ship company data easily. But compared with relational database, the efficiency of processing query for XML document is a serious issue. There are various researches surveyed about efficient XML query processing; they can be classified into the following three categories: 1) Path indexing ${ }^{(2)}$, creates a path summary from XML data. Path indexing speeds up the evaluation of single path queries. 2) Node indexing ${ }^{(3)}$, indexes each data node by some numbering schemas. 3) Sequence-based indexing (4), transforms both XML documents and queries into sequence, and evaluates queries based on sequence matching. Although there are so many efficient querying methods, the basic XML document deployment is not discussed . If the duplication of dataset is not deployed properly, its performing efficiency could be reduced to a lower state. In order to reduce this disadvantage, a proper redesigned data structure should be considered.

In the maritime field, the industry is involved with several different government levels (such as procedures of arriving in, cargo clearance, immigration declaration, etc), and each department has its own specific requirement for desired information, so finally the total data storage capacity that is needed could be very huge. At the same time, the duplication of similar data could be very high. For example, the same or similar cargo carried

\footnotetext{
* student member Kobe University (T658-0022 5-1-1 Fukae-minamimachi, Higashinada-ku, Kobe, Japan) hiroshi@cs.maritime.kobe-u.ac.jp

** member Kobe University waka@cs.maritime.kobe-u.ac.jp

$* * * \quad$ member Kobe University yam@maritime.kobe-u.ac.jp

$* * * * \quad$ student member Kobe University harada@cs.maritime.kobe-u.ac.jp
} 
by a container ship could be deployed in different blocks within the ship or the container yard. In this case, most of the cargo data set could be duplicated except for deploying locations. This kind of data set with high duplication could seriously affect its query efficiency if the data structure is not properly restructured.

For the application of our restructuring data structure, we apply the XML data model on containers. Because of the high duplication of this data set, an original XML document could be restructured to the more proper indexed XML document, and then we use XML Path Language (XPath) ${ }^{(5)}$ to verify that the querying efficiency is improved.

This paper is organized as follows; section 2 introduces our XML data model definition applied on containers and presents our XML document restructuring mechanism. In Section 3 we present experimental results which show the effectiveness of our proposed data structure. Finally Section 4 summarizes the contributions of this paper.

\section{XML Data Model for Container Operation}

Container operation is the Unit Load System which uses containers as units for unifying cargo transportation. Since container operation was introduced in 1957, it became an important subject for port transportation.

In this paper, we focus on port container operation, establish the cargo XML data model for container management by treating a single container as a XML data node. The container yard is on the top as the root node, multiple ship companies are below it, and each ship company transports many containers. We call this original container XML data model as original container XML data model and illustrate its structure in Fig. 1.

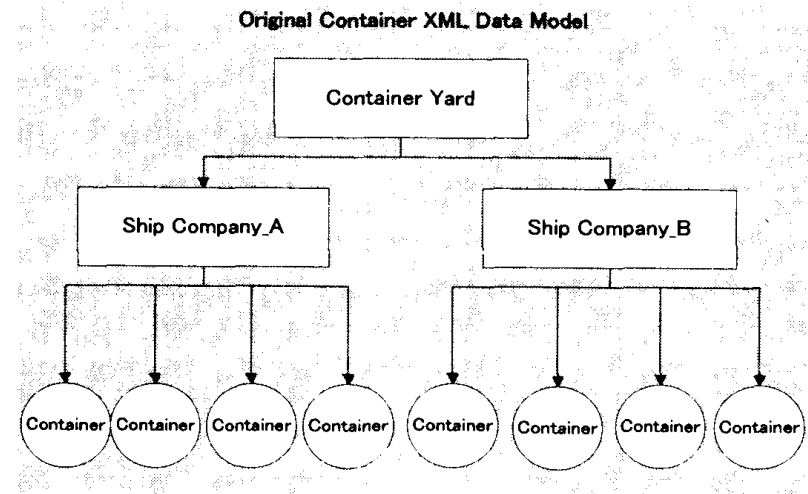

Fig. 1 Original container XML data model
The detail of container XML data model will be given in next section. This data model is built for verifying the efficiency of querying experiments but not proposing the actual container operation implementation.

\subsection{Container XML Data Model Definition}

In order to applying the container yard management, data node for single container should be composed of multiple information. Based upon the Customs clearance procedures in place, we collect the desired information as the attributes for containers shown in Table 1 .

Table 1 Desired container attributes

\begin{tabular}{|c|c|}
\hline Attribute & Description \\
\hline Serial number & $\begin{array}{l}\text { Identification ruled by ISO standard for } \\
\text { each container. }\end{array}$ \\
\hline Cargo context & $\begin{array}{l}\text { Classification of Full Container Load } \\
\text { Cargo (FLC) and Less than Container } \\
\text { Load Cargo (LCL). }\end{array}$ \\
\hline Ship company & $\begin{array}{l}\text { The ship company having responsibility } \\
\text { for cargo transportation. }\end{array}$ \\
\hline Gross weight & $\begin{array}{l}\text { Cargo total weight (including container } \\
\text { tare weight). }\end{array}$ \\
\hline Port of departure & The port that containers were loaded. \\
\hline Port of destination & The port that container will be unloaded. \\
\hline Dangerous cargo & $\begin{array}{l}\text { Cargo which, because of its dangerous } \\
\text { properties, is subject to special regulations } \\
\text { for its transport. }\end{array}$ \\
\hline Place of origin & The place of cargo production. \\
\hline Name of party & The name of cargo owner. \\
\hline Kind of package & The classification of cargo. \\
\hline Description & Description of cargo context. \\
\hline Container type & $\begin{array}{l}\text { Containers can be classified as several } \\
\text { types by their purposes (dry, flat, opentop, } \\
\text { bulk, reefer, tank, ventilated and pen). }\end{array}$ \\
\hline
\end{tabular}

According to our definition for container node, the XML Schema for original container XML data model can be illustrated as Fig. 2. Each node under container node is a leaf node which carries text or numeric information. Fig. 3 is some simple entity of the complete original XML data model as an example. 


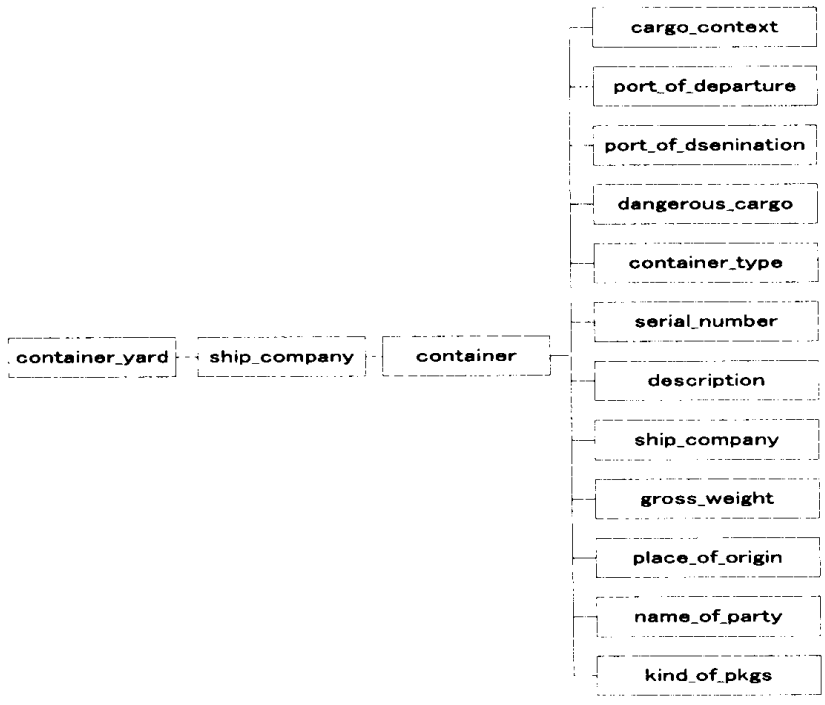

Fig. 2 Original container XML data model Schema

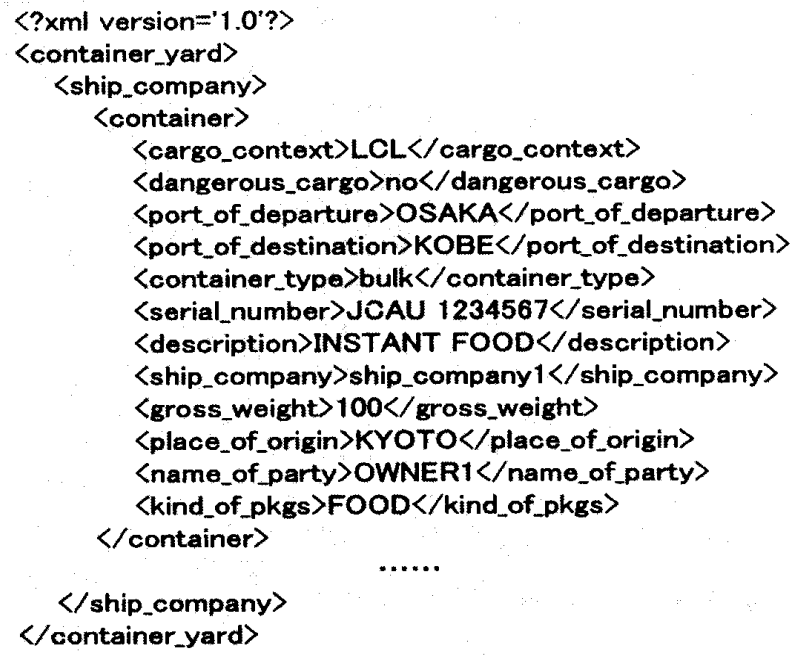

Fig. 3 Original container XML document sample

\subsection{Restructuring Container XML Data Model}

The XML standard defines that an XML document has to be a nested structure and only the leaf elements (the lowest layer elements) can store strings or numeric information. Because of this essential limitation, XML documents have more redundant context and need more storage space than relational database. This fact directly affects the efficiency of querying.

For one container, it has various attributes and each of those attributes has the one-to-one relationship with the container. But if we see the entire data structure, there are still some attributes with high duplication which can be grouped. Let's take the "dangerous cargo" attribute of the container data model as an example, its value can be just "yes" or "no". But once the "dangerous cargo" attribute is defined within the XML schema, the instance of it has to occur repeatedly within each single container node of XML document. So if we can group the attributes with high duplication within XML documents, then we can reduce the data storage space effectively.

Although the elements of XML document (except leaf elements) cannot store information, it is available to self-define multi attributes for every element. By using this function, we can translate highly duplicated attributes with finite quantity of leaf elements into the self-defined attribute values of upper layer elements. A translation sample can be given as Fig. 4.

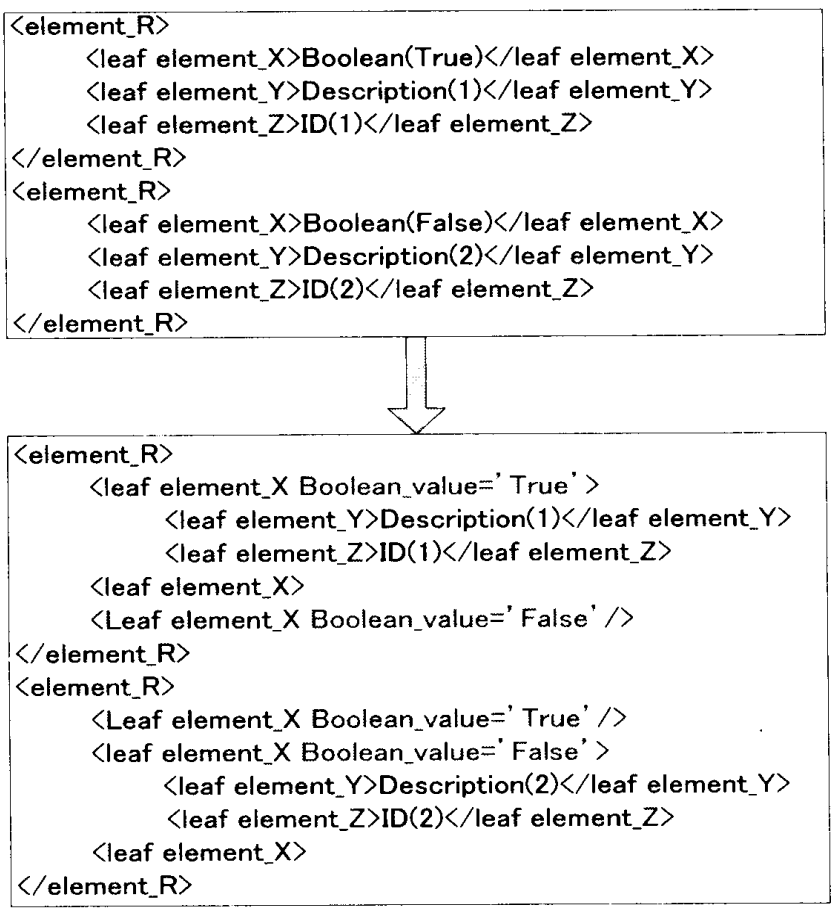

Fig. 4 XML document restructuration sample

For our original container XML data model, we can collate the attributes with high duplication as index group and restructure the original XML document into an indexed XML document. The detail of index group is given in Table 2.

Table 2 Index group

\begin{tabular}{|c|c|c|}
\hline Attributes & Possible values & Quantity \\
\hline Cargo context & FCL, LCL & 2 \\
\hline Dangerous cargo & Yes, no & 2 \\
\hline Port of departure & PL1, PL2, PL3,PL4, PL5 & 5 \\
\hline Port of destination & PD1, PD2, PD3,PD4, PD5, PD6 & 6 \\
\hline Container type & $\begin{array}{c}\text { dry, flat, opentop, bulk, reefer, } \\
\text { tank, ventilated pen }\end{array}$ & 8 \\
\hline
\end{tabular}


In order to improve the querying efficiency, we restructure the indexed XML document by the order of attribute quantity as shown in Table. 2.

A sample of the indexed container XML document is illustrated as Fig. 5.

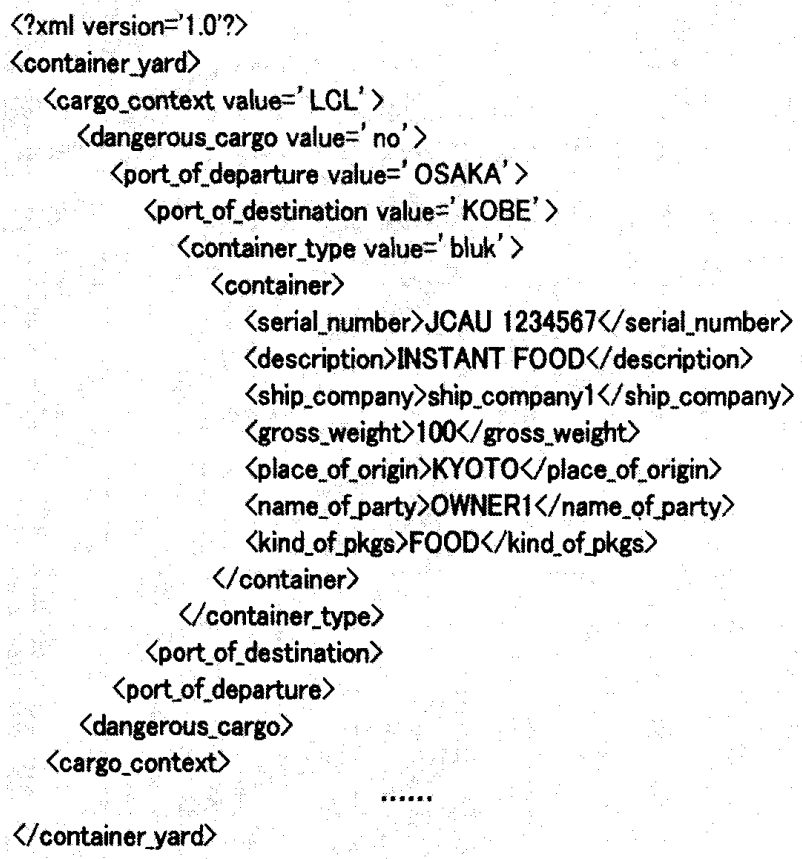

Fig. 5 Indexed container XML document sample

Each container node within Original XML container document has 12 leaf elements. In our test case, we adopt 5 leaf elements from them as the index attributes to process the grouping procedure and each container within the Indexed container XML document only has 7 leaf elements. So everytime when a new container is added, the data difference between the Original XML container document and the Index container XML document will increase 5 . On the other hand, the subject varities of the index group are fixed according to the number of index attributes at the first place. Therefore the data capacity will totally decrease along with the increasement of the number of containers.

In order to verify the effect of data storage space, we made several cases of different total container number from 1,000 to 500,000 for both original and indexed XML documents. For the first 1,000 container case, the file storage space reducing percentage is $83.4 \%$. And along with the growth of container number, finally the file storage space reducing percentage reaches to $74.0 \%$.

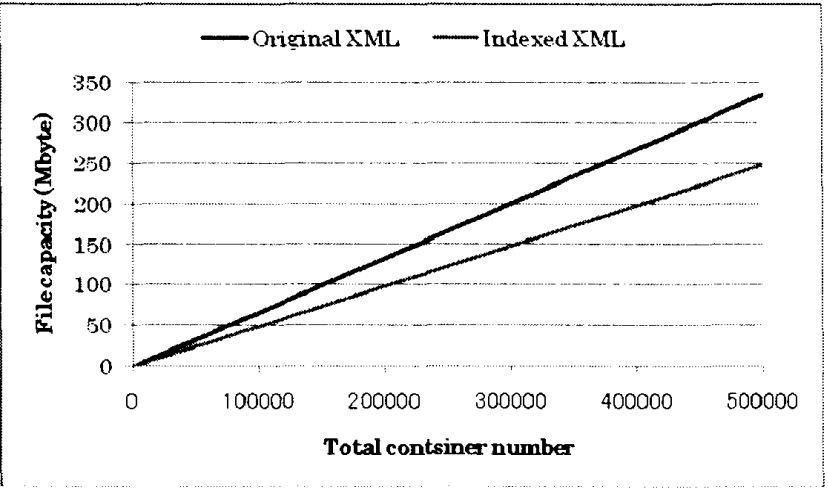

Fig. 6 XML files capacity comparison

\section{Performance Evaluation}

In order to verify the efficiency of our XML data structure, we use visual basic.net 2008 express version to build an XML document generator and XPath query application.

Our XML document generator can establish random original and restructured XML documents by designating total container number. The important index attributes of each container are assigned within the default candidates. For example, the value of container type can only be one of dry, flat, opentop, bulk, reefer, tank, ventilated and pen. The other attributes are given as strings, integers or their combination randomly. The original and indexed container XML datasets with 100,000 containers could be built within one minute.

The XPath query function can process single path query (direct input XPath) and scheduled query procedures (default 10 constructions we designed). Each query procedure runs 20 times to get their average and ensure that querying results can be more accurate.

Performance experiments were run on a $2 \mathrm{GHz}$ PC-compatible machine with RAM of $1 \mathrm{~GB}$ running Windows XP.

\subsection{XML test datasets characteristics}

In the test datasets to use for performance evaluation, we set the total container number as one hundred thousand. Table 3 shows the properties of two datasets, original and indexed container XML documents. After the restructuring procedure, the dataset capacity, line number and element number reduces to $73.7 \%, 70.7 \%$ and $68.8 \%$ respectively. We can see that the restructuring procedure effectively reduce the storage space from the results. 
Table 3 Characteristics of test datasets

\begin{tabular}{|c|c|c|}
\hline 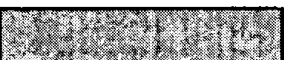 & Originar xal & W mated Xavi \\
\hline Size (MB) & 63.2 & 46.6 \\
\hline Max depth & 3 & 7 \\
\hline Line\# & $1,700,033$ & $1,202,216$ \\
\hline Element\# & $1,600,016$ & $1,101,107$ \\
\hline
\end{tabular}

\subsection{XML Path Language}

$\mathrm{XPath}$ is a language for addressing parts of an XML document. It also provides basic facilities for manipulation of strings, numbers and booleans.

XPath doesn't treat an XML document as strings set, but use XML parser to analyze XML tree structure. The target that XPath points to is the set of nodes composed of elements, attributes and texts within XML documents.

For example, with the root node as context node, the location path "News/Item[@date]" selects all the news elements with a date attribute. An example of XPath query is shown as Fig. 7. In this example, we use XPath construction as “/News/Item[@date='080521']” to query the XML document. The query result returned would be a node set collection composed of node (Headline_a) and node (Headline_b), then we can use various node set functions to get the contexts of the result node set.

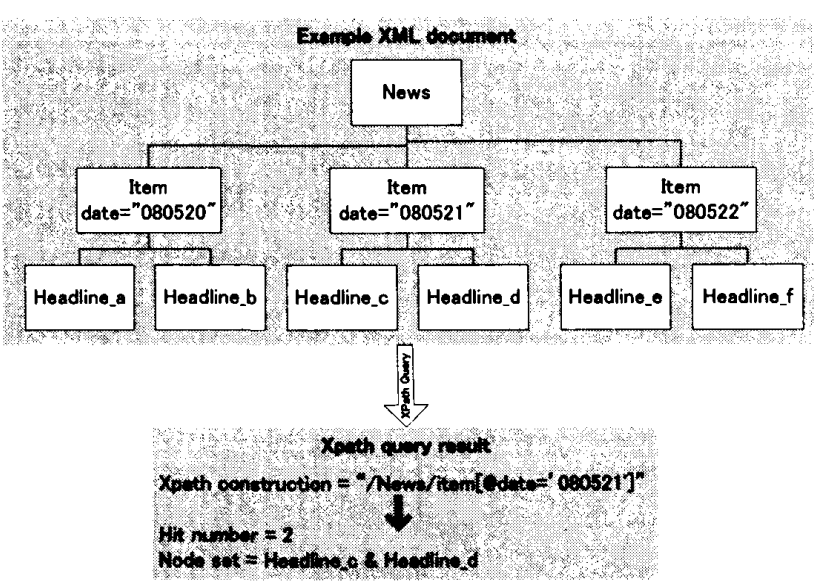

Fig. 7 XPath query example

The common XPath constructions can be given as Table 4.
Table 4 Common XPath constructions

\begin{tabular}{|c|c|c|}
\hline Futl construction & $\begin{array}{l}\text { Simplified } \\
\text { construction }\end{array}$ & \multicolumn{1}{|c|}{ Description } \\
\hline child:: & Ignored & $\begin{array}{l}\text { Child node of construction } \\
\text { node }\end{array}$ \\
\hline attribute:: & $@$ & $\begin{array}{l}\text { Attribute node of } \\
\text { construction node }\end{array}$ \\
\hline $\begin{array}{c}\text { /descendant-or- } \\
\text { self::node()/ }\end{array}$ & $/ /$ & $\begin{array}{l}\text { Construction itself and its } \\
\text { descendant nodes }\end{array}$ \\
\hline self::node() &. & Construction node itself \\
\hline parent::node() &.. & $\begin{array}{l}\text { Parent node of construction } \\
\text { node }\end{array}$ \\
\hline child::text() & text() & $\begin{array}{l}\text { All text belong to the child } \\
\text { nodes of construction node }\end{array}$ \\
\hline
\end{tabular}

In this paper, we use XPath constructions to verify the efficiency for querying the original and restructured XML documents.

\subsection{Experimental results}

We design 10 query missions for evaluation test with different purposes for our data sets shown in Table 5 .

Table 5 Sample queries for data sets

\begin{tabular}{|c|c|c|}
\hline 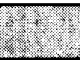 & Description & Answer" \\
\hline \multirow{2}{*}{ Q1 } & Original: //container & \multirow{2}{*}{100,000} \\
\hline & Indexed: //container & \\
\hline \multirow{2}{*}{ Q2 } & Original: //container[serial_number='986'] & \multirow{2}{*}{1} \\
\hline & Indexed: //container[serial_number='986'] & \\
\hline \multirow{2}{*}{ Q3 } & Original: sum(//container/gross_weight/text()) & \multirow{2}{*}{1} \\
\hline & Indexed: sum(//container/gross_weight/text()) & \\
\hline \multirow[b]{2}{*}{ Q4 } & Original: //container[cargo_context='LCL'] & \multirow[b]{2}{*}{49,971} \\
\hline & $\begin{array}{l}\text { Indexed: } \\
\text { //cargo_context[@value='LCL']//container }\end{array}$ & \\
\hline \multirow[b]{2}{*}{ Q5 } & Original: //container[port_of_departure='PL3'] & \multirow[b]{2}{*}{20,112} \\
\hline & $\begin{array}{l}\text { Indexed: } \\
\text { //port_of_departure[@value='PL3']//container }\end{array}$ & \\
\hline \multirow[b]{2}{*}{ Q6 } & $\begin{array}{l}\text { Original: } \\
\text { //container[port_of_destination='PD4'] }\end{array}$ & \multirow[b]{2}{*}{16,841} \\
\hline & $\begin{array}{l}\text { Indexed: } \\
\text { //port_of_destination[@value='PD4']//containe } \\
\mathrm{r}\end{array}$ & \\
\hline \multirow[b]{2}{*}{ Q7 } & Original: //container[container_type $=$ 'reefer'] & \multirow[b]{2}{*}{12,372} \\
\hline & $\begin{array}{l}\text { Indexed: } \\
\text { //container_type[@value='reefer']//container }\end{array}$ & \\
\hline \multirow[b]{2}{*}{ Q8 } & Original: //container[dangerous_cargo $=$ 'yes'] & \multirow[b]{2}{*}{49,940} \\
\hline & $\begin{array}{l}\text { Indexed: } \\
\text { //dangerous_cargo[@value='yes']//container }\end{array}$ & \\
\hline Q9 & & 6,204 \\
\hline
\end{tabular}




\begin{tabular}{|c|c|c|}
\hline & $\begin{array}{l}\text { //container[container_type }={ }^{\prime} \text { 'ventilated' and } \\
\left.\text { cargo_context }={ }^{\prime} F C^{\prime}\right]\end{array}$ & \\
\hline & $\begin{array}{l}\text { Indexed: } \\
\text { //cargo_context[@value='FCL']//container_typ } \\
\text { e[@value='ventilated']//container }\end{array}$ & \\
\hline \multirow[b]{2}{*}{ Q10 } & $\begin{array}{l}\text { Original: //container[dangerous_cargo }=\text { 'yes' } \\
\text { and port_of_destination='PD6' and } \\
\text { port_of_departure='PL2'] }\end{array}$ & \multirow[b]{2}{*}{1,646} \\
\hline & $\begin{array}{l}\text { Indexed: } \\
\text { //dangerous_cargo[@value='yes']/port_of_dep } \\
\text { arture[@value='PL2']/port_of_destination[@v } \\
\text { alue='PD6']//container }\end{array}$ & \\
\hline
\end{tabular}

The performance of the querying procedures is illustrated in Fig. 8.

Q1 queries for the total container number, so it only search until the element "container". For the original XML dataset, it would be the third layer. And for the indexed XML dataset, it would be the seventh layer. But both of them stop searching for the below various attributes, so this query doesn't spend so much time. Because indexed XML dataset has a smaller capacity than original XML dataset, so its efficiency is still a little better than original XML dataset.

Q2 queries for a certain container among all containers. As it designate a specific container serial number, this query has to search until the lowest layer to check the serial number attribute value. For original XML dataset, this query would need to check about 1.2 million elements. That's why compared with Q1, Q2 spend ten times of time for querying. According to the result, indexed XML dataset is still a little better than original XML dataset.

Q3 requests for the sum of all containers' gross weight. Like Q2, it has to search all attributes under element "container". But compared with Q2, indexed XML dataset outperform original XML dataset much better than $\mathrm{Q} 2$. The reason is the difference of leaf elements' number between these two datasets. The leaf element number under original XML dataset is 12 , and indexed XML dataset is 7 . This difference effectively reduces the querying time.

Q4 through Q8 query for the containers belong to a specific catalog. In these cases, the efficiencies of indexed XML datasets are greatly outperform original XML datasets.

Q9 queries for containers belong to two specific catalogs; Q10 queries for containers belong to three specific catalogs. According to the results, we can see that their performance improvements are even better than Q4 through Q8.

From the experiment results, we can see that the performances of our indexed XML dataset significantly outperform the original XML dataset. If the request construction contains more grouped index attributes simultaneously, our indexed method will be able to achieve more performance to improve querying efficiency.

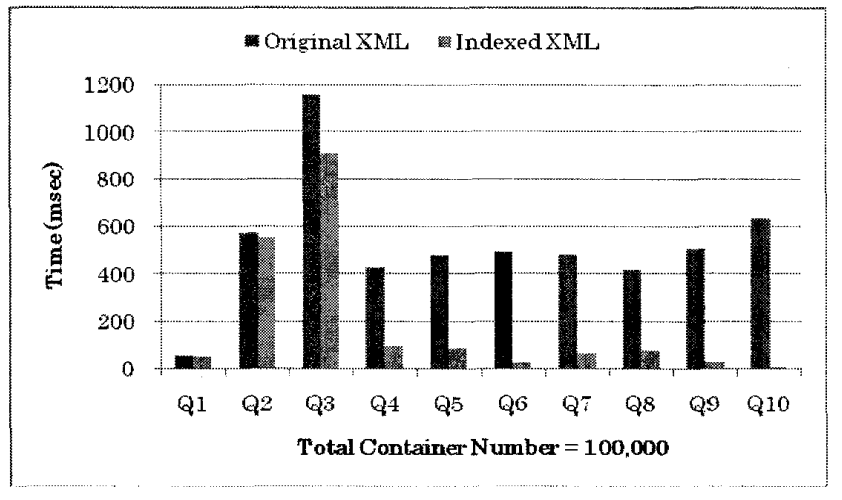

Fig. 8 Performance comparison of original and indexed XML documents for XPath querying

\section{Conclusion}

As maritime industry used to perform its operations not depending heavily on information technologies, it seems that it has not paid more attention on the issue for query efficiency. But compared with the capability of cargo tracking for overland logistic system, sea transportation system still cannot achieve such kind of operation precision. Manual management is no longer the point in B2B generation, but most of complex processes should be handled by servers automatically. Along with the growth of applications implemented for maritime field, data storage capacity and single query efficiency will affect entire system performance explicitly.

In this paper, we propose a mechanism to group the XML document elements by using attributes as leaf element value. By this mean, we can reduce the storage space for XML document effectively. At the same time, with increase in duplication of dataset, we can gain more efficient performance.

Our research focus on the improvement of data query efficiency for now. The future objective is using this 
efficient data structure as the fundament to apply further researches and implementations on various levels.

\section{References}

(1) Chia-Hung Shih, Nobukazu Wakabayashi, and Saburo Yamamura, A Distributed Data Model for Port Administration and On Board Information and Service Management, Japan Institute Navigation, pp. 65-72, 2008.3.

(2) R. Goldman and J. Widom, Dataguides: Enabling query formulation and optimization in semistructured databases, VLDB, 1997.

(3) Q. Li and B. Moon, Indexing and querying XML data for regular path expression, VLDB, 2001.

(4) Qinghua Zou, Shaorong Liu, and Wesley W.Chu, Ctree: A Compact Tree for Indexing XML Data, Workshop On Web Information And Data Management, pp. 39-46, 2004.

(5) XML Path Language (XPath) Version 1.0, W3C Recommendation, 16 November 1999.

\section{Questions and Answers}

Tasseda EL HOCINE (Tokyo University of Marine

Science and Technology):

(1) Why do you use the XML documents to store data instead of relational database engine?

(2) What backup do you provide for your stored data?

\section{Chia-Hung SHIH:}

(1) Although relational database is a very powerful method for data storage, XML is growing in a high speed recently because of its various characteristics that relational database doesn't have. In $\mathrm{B} 2 \mathrm{~B}$ generation, communications between servers automatically is a more important issue than efficiency. XML can achieve some objectives with its semantic structures that relational database is not able to meet by sacrificing some efficiency.

(2) Not like relational database, XML is not combined by a specific system like SQL Server. When XML is treated as a method of data storage, it is just a simple text-format file. So we can easily backup it by simple copy operation or even upload it to a FTP server if the internet is available.

Junji FUKUTO (National Maritime Research Institute):

(1) Could you explain merits for using XML database for data storage and for data handling system?

\section{Chia-Hung SHIH:}

(1) XML has several important characteristics that relational database doesn't have which can be described below:

- Tree architecture: The unstructured data that relational database is difficult to handle can be translated to the Semi-structured data by definitions of DTD (Document Type Definition) or XML Schema.

- Self-define tag: XML provides a markup structured data construction; it allows users to define specific professional field tags by themselves.

- Independency of data structure: data is separated with display format. Because the content and display format are separated, it is much easier to read and maintain for both human beings and computers.

These characteristics make XML be able to exchange data between servers automatically without human judgments. More details are given in our pervious propose paper ${ }^{(1)}$. 